\title{
Current Proportion of Women Receiving Perinatal Psychosocial or Psychological Intervention in Japan
}

\author{
Shunji Suzukia, b, c, Yasushi Kuribayashi ${ }^{\mathrm{b}}$, Hideo Matsuda ${ }^{\mathrm{b}}$, Yasuyuki Asakawa ${ }^{\mathrm{b}}$, Akihiko Sekizawa $^{\mathrm{b}}$, \\ Masanobu Tanaka ${ }^{\text {b }}$, Takashi Okai ${ }^{b}$, Katsuyuki Kinoshita ${ }^{b}$
}

\begin{abstract}
Background: In this study, we examined the current status of psychosocial or psychological intervention for women during pregnancy and the postpartum period in Japan.

Methods: We estimated the number of women receiving perinatal psychosocial or psychological intervention in Japan. On December 2015, we requested 2,462 obstetrical facilities that are members of the Japan Association of Obstetricians and Gynecologists (JAOG) to provide information on women who received psychosocial or psychological intervention during pregnancy, the hospitalization period for childbirth and the puerperal 1 month in 2014. A total of 1,305 (53.0\%) of the 2,462 obstetrical facilities responded with valid information on a total of 515,373 women, accounting for approximately $52 \%$ of all deliveries that occurred in Japan during the study period.
\end{abstract}

Results: The number of women who received psychosocial or psychological intervention during pregnancy, the hospitalization period for childbirth and the puerperal 1 month were 4,843 (0.94\%), 4,791 $(0.93 \%)$ and $3,015(0.59 \%)$, respectively. In total, 8,743 women $(1.70 \%)$ received psychosocial or psychological intervention in 2014.

Conclusion: Considering the response rate, the number of women requiring perinatal psychosocial or psychological intervention was estimated to be 16,000 per year in Japan.

Keywords: Psychosocial or psychological intervention; Pregnancy; Postpartum; Japanese women

\section{Introduction}

Recently, perinatal mental disorders have become significant complications of pregnancy and the postpartum period $[1,2]$.

\footnotetext{
Manuscript accepted for publication April 20, 2016

aJapanese Red Cross Katsushika Maternity Hospital, Tokyo, Japan

bJapan Association of Obstetricians and Gynecologists, Tokyo, Japan

${ }^{c}$ Corresponding Author: Shunji Suzuki, Department of Obstetrics and Gynecology, Japanese Red Cross Katsushika Maternity Hospital, 5-11-12 Tateishi, Katsushika-ku, Tokyo 124-0012, Japan. Email: czg83542@mopera.ne.jp
}

doi: http://dx.doi.org/10.14740/jocmr2568w
Perinatal mental disorders impair a woman's function and are associated with the suboptimal development of her children. Perinatal mental health care is required for the emotional wellbeing of pregnant women and their children, partners and families. The early detection and effective management of perinatal mental disorders are critical for the welfare of women and their children [3]. For example, women who receive psychosocial or psychological intervention have been observed to be significantly less likely to develop postpartum depression compared with those receiving standard care [3]. There may be a number of steps in perinatal psychosocial or psychological intervention to help remain emotionally balanced during pregnancy and after childbirth. Promising interventions include the provision of intensive, professionally-based postpartum home visits, telephone-based peer support, and interpersonal psychotherapy. These interventions have been performed in some regions of Japan and they have been reported to decrease the incidence of postpartum depression [4].

In this study, based on this background, we examined the current status of psychosocial or psychological intervention for women during pregnancy and the postpartum period in Japan.

\section{Methods}

We estimated the number of women receiving perinatal psychosocial or psychological intervention in Japan based on a questionnaire survey. In addition, we also examined the regional disparities of the implementation rate of intervention (Fig. 1). The protocol for this survey was approved by the Ethics Committee of the Japan Association of Obstetricians and Gynecologists (JAOG).

On December 2015, we requested 2,462 obstetrical facilities that are members of JAOG to provide information on women who received psychosocial or psychological intervention during pregnancy, the hospitalization period for childbirth and the puerperal 1 month in 2014. A total of 1,305 (53.0\%) of 2,462 obstetrical facilities responded with valid information on a total of 515,373 women, accounting for approximately $52 \%$ of all deliveries that occurred in Japan during the study period (approximately 1,008,000 births).

\section{Results and Discussion}

The number of women who received psychosocial or psy- 


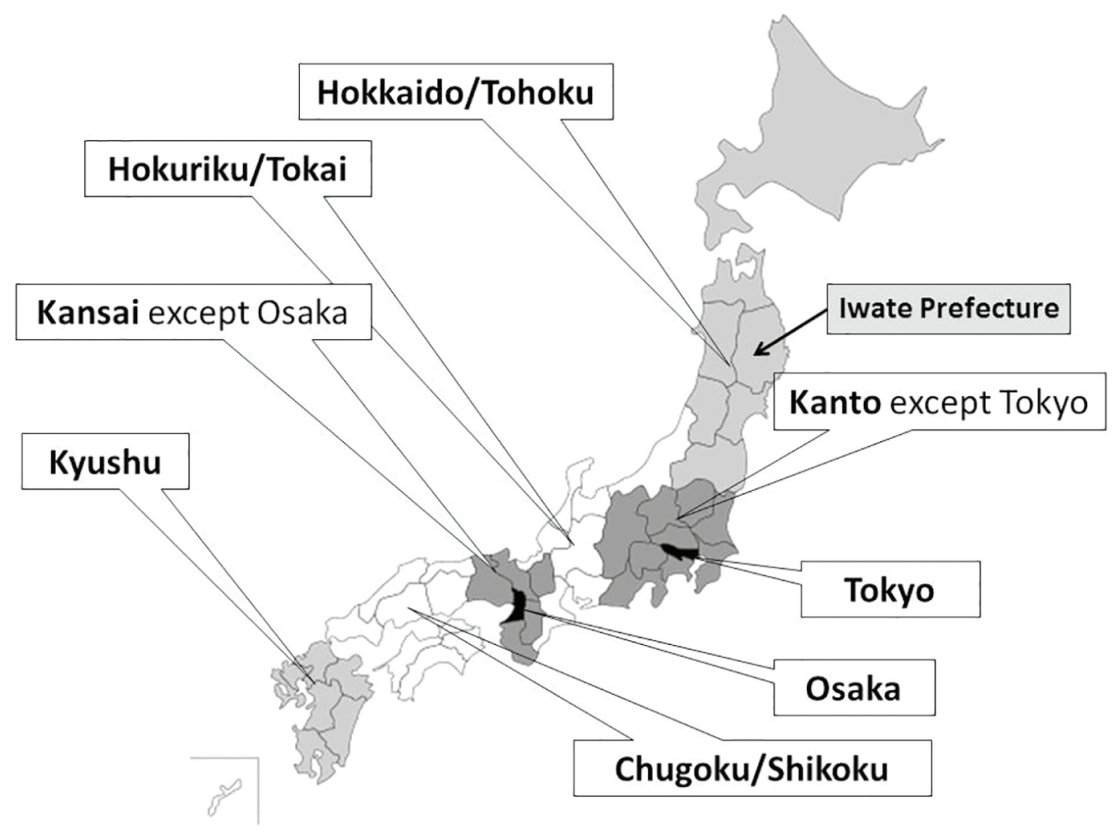

Figure 1. Regional disparities of the implementation rate of intervention.

chological intervention during pregnancy, the hospitalization period for childbirth and the puerperal 1 month were 4,843 $(0.94 \%), 4,791(0.93 \%)$ and $3,015(0.59 \%)$, respectively. In total, there were 8,743 women $(1.70 \%)$ who received psychosocial or psychological intervention in 2014. Considering the response rate, the number of women requiring perinatal psychosocial or psychological intervention was estimated to be 16,000 per year in Japan.

Table 1 shows the distribution of women who received perinatal psychosocial or psychological intervention in Japan. There were significant regional disparities in the implementation rate of intervention in Japan. For example, in Iwate Prefecture in the Hokkaido and Tohoku region, the rate of women who received the intervention was high (3.01\%). In Iwate Prefecture, the active child-rearing support based on the prenatal and postpartum mental health evaluation has been reported to be carried out in cooperation between medical staff and the prefectural government [4]. In this region, in addition, mental disorders associated with the Great East Japan Earthquake are still being followed in women [5]. While in Osaka, one of the metropolises of Japan, the rate of women received the intervention was not high $(1.33 \%)$. In Osaka, the rate of pregnant women with socio-economic problems such as a low income and no prenatal examination has been suggested to be higher than that in other prefectures of Japan according to Japanese media reports and the Osaka Homepage $[6,7]$. The socio-economic problems have been observed to be associated with the increased risk of women's mental disorders [8], and robust social support systems for such women have been implemented in Osaka [6, 7]. In Osaka, therefore, the development of perinatal mental disorders may not mainly be due to socio-economic problems; however, there is a possibility that the importance of perinatal mental health care is not widely recognized in Osaka, although some of the facilities are conceived about the matter [7]. Based on these possibilities, the current proportions of women receiving perinatal psychosocial or psychological intervention may not necessarily correlate with those of women complicated by mental disorders. In any case, adequate perinatal mental health care must be provided that has been adapted to the situation of each region in Japan.

\section{Conclusions}

The number of women requiring perinatal psychosocial or psychological intervention was estimated to be 16,000 per year in Japan. However, there are significant regional disparities in perinatal psychosocial or psychological intervention in Japan.

\section{Grant Support}

This work was supported in part by a Grant-in-Aid for Scientific Research from the Japanese Ministry of Health, Labour and Welfare.

\section{Conflicts of Interest}

The authors declare that there are no conflicts of interest regarding the publication of this paper.

\section{Author Contributions}

SS contributed to the research design, analyzed data and wrote the manuscript. YK, YM and YA analyzed data and contrib- 
Table 1. Distribution of Women Receiving Perinatal Psychosocial or Psychological Intervention in Japan

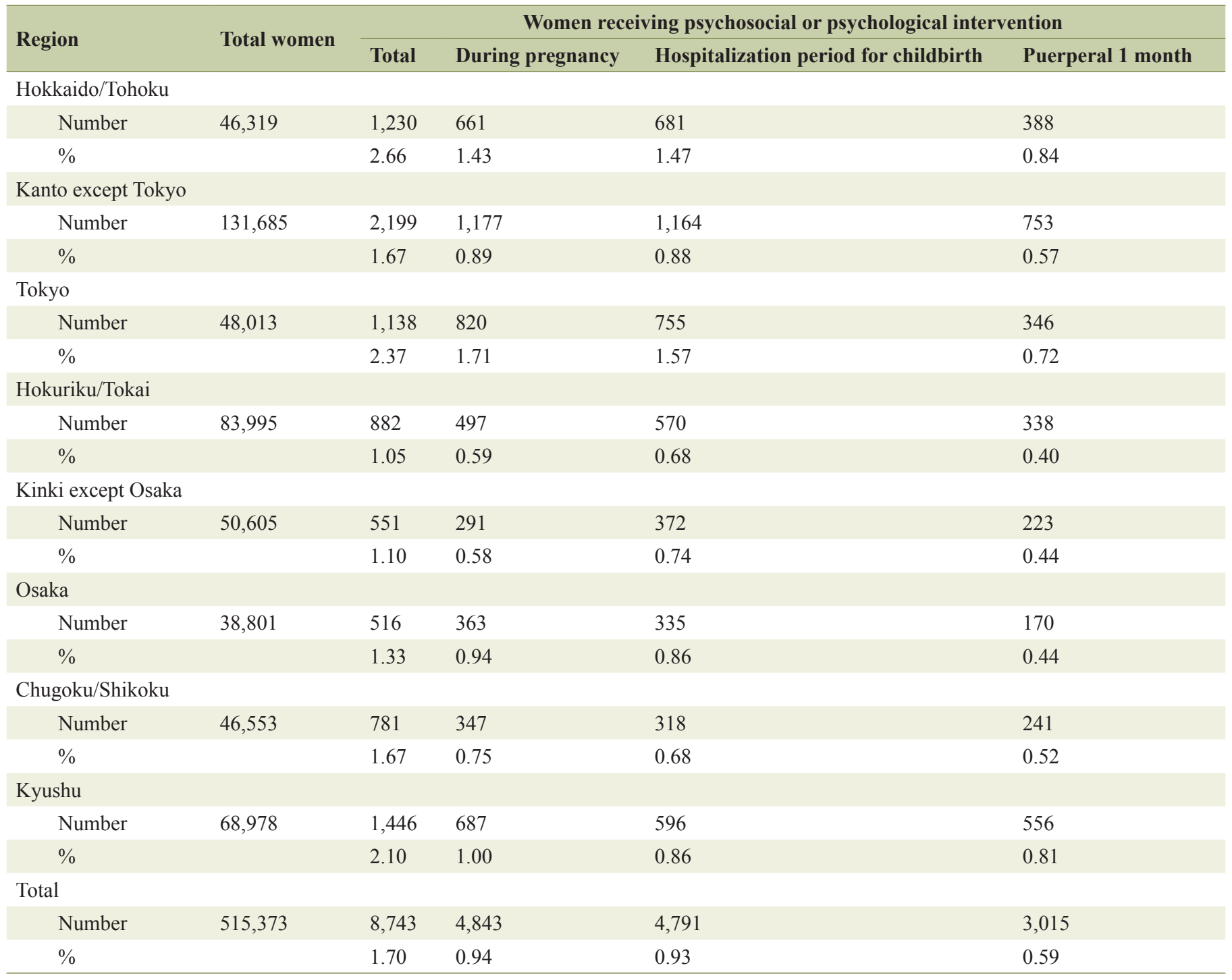

uted to the manuscript. AS, MT, TO and KK contributed to the research design and manuscript.

\section{References}

1. O'Hara MW, Wisner KL. Perinatal mental illness: definition, description and aetiology. Best Pract Res Clin Obstet Gynaecol. 2014;28(1):3-12.

2. St. Johna Beat Baby Blues. Perinatal \& Postnatal Depression Support. http://www.beatbabyblues.com.au/ (March 24, 2016).

3. Dennis CL, Dowswell T. Psychosocial and psychological interventions for preventing postpartum depression. Cochrane Database Syst Rev. 2013;2:CD001134.

4. Yoshida K. Trends and Future Prospects of maternal mental health (in Japanese). Report of the scientific re- search in the Japanese Ministry of Health, Labour and Welfare (2012). http://www.aiiku.or.jp/ doc/houkoku/ h24/19025A060.pdf (March 24, 2016).

5. Nishigori H, Sasaki M, Obara T, Nishigori T, Ishikuro M, Metoki H, Sugawara J, et al. Correlation Between the Great East Japan Earthquake and Postpartum Depression: A Study in Miyako, Iwate, Japan. Disaster Med Public Health Prep. 2015;9(3):307-312.

6. Osaka Prefectural government: Application situation of welfare (in Japanese). http://www.city.osaka.lg.jp/fukushi/page/0000086901.html (March 24, 2016).

7. Osaka Prefectural government: Survey of women without prenatal examination (in Japanese). http://www.pref. osaka.lg.jp/kenkozukuri/boshi/mijyusin.html (March 24, 2016).

8. Suzuki S. Economic problems and mental disorders in Japanese pregnant women. J Clin Med Res. 2015;7(5):367. 